\title{
3D Printed Biodegradable Polyurethaneurea Elastomer Recapitulates Skeletal Muscle Structure and Function
}

\author{
Seyda Gokyer ${ }^{1}$, Emel Yilgor ${ }^{2}$, Iskender Yilgor ${ }^{2}$, Emine Berber $^{3}$, Engin Vrana ${ }^{3,4}$, Kaan \\ Orhan $^{5,8}$, Yanad Abou Monsef ${ }^{6}$, Orcun Guvener ${ }^{7}$, Murat Zinnuroglu ${ }^{8}$, Cagdas Oto ${ }^{7,9}$, \\ Pinar Yilgor Huri ${ }^{1,9 *}$
}

\footnotetext{
${ }^{1}$ Ankara University Faculty of Engineering, Department of Biomedical Engineering, Ankara, Turkey ${ }^{2}$ KUYTAM Surface Science and Technology Center, Koç University Department of Chemistry, Istanbul, Turkey

${ }^{3}$ National Institute of Health and Medical Research, INSERM UMR1121, Biomaterials and Bioengineering, 11 Rue Humann, 67000, Strasbourg, France

${ }^{4}$ Spartha Medical, 14B Rue de la Canardiere 67100, Strasbourg, France

${ }^{5}$ Ankara University Faculty of Dentistry, Department of Dentomaxillofacial Radiology, Ankara, Turkey

${ }^{6}$ Ankara University Faculty of Veterinary Medicine, Department of Pathology, Ankara, Turkey

${ }^{7}$ Ankara University Faculty of Veterinary Medicine, Department of Anatomy, Ankara, Turkey

${ }^{8}$ Gazi University Faculty of Medicine, Department of Physical Medicine and Rehabilitation, Ankara, Turkey

${ }^{9}$ Ankara University Medical Design Research and Application Center MEDITAM, Ankara, Turkey
}

*Correspondence to: Assoc. Prof. Dr. Pinar Yilgor Huri (E-mail: phuri@ankara.edu.tr) 


\section{$\underline{\text { Supporting Information }}$}

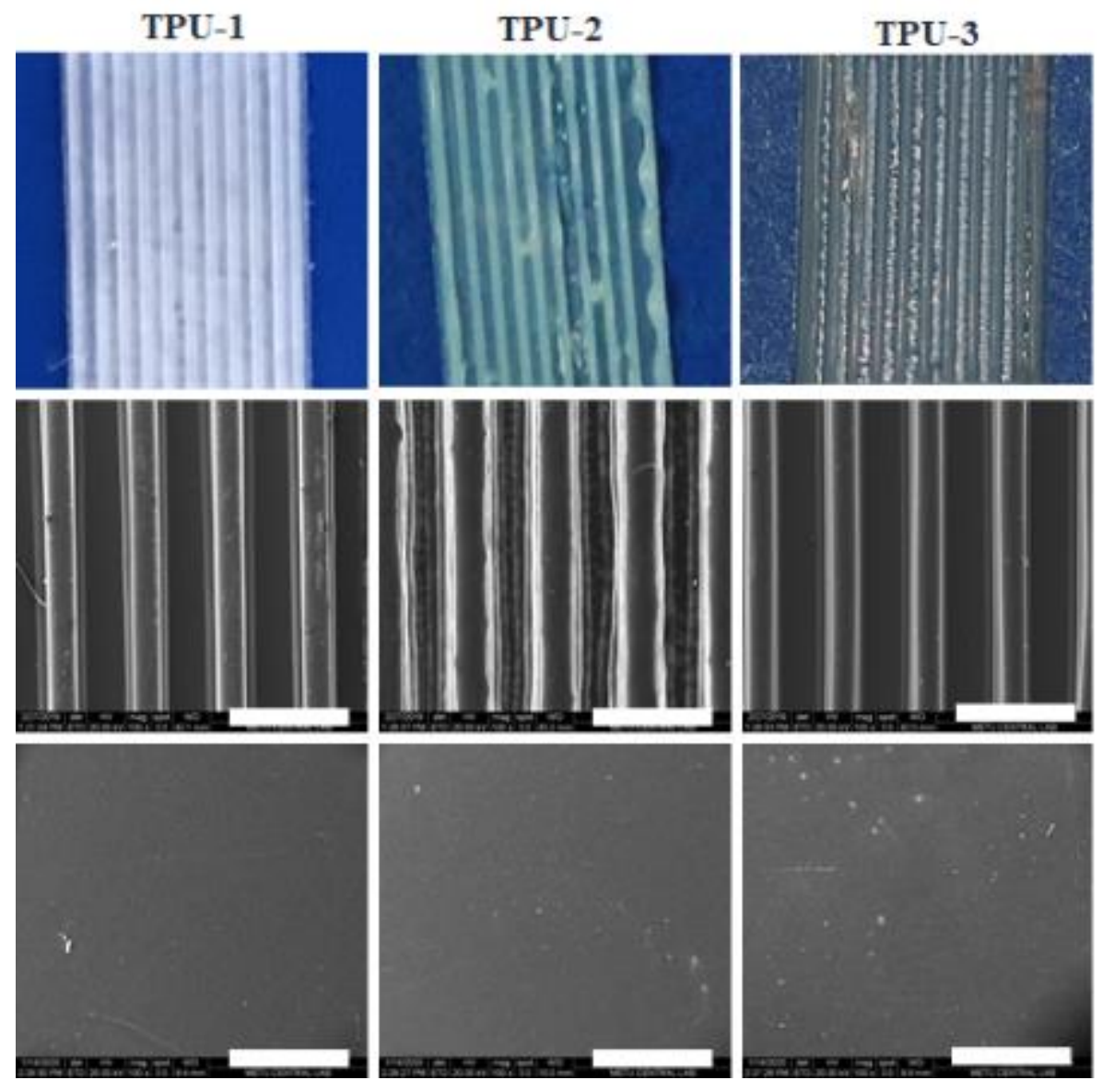

Figure S1-Sn. Gross morphology pictures (upper row), SEM micrographs of 3D printed parallel-aligned scaffolds (middle row), and solvent cast smooth films (bottom row) produced from TPU-1, TPU-2 and TPU-3 in solution state. Scale bars show $1 \mathrm{~mm}$ in the SEM micrographs. 
FTIR
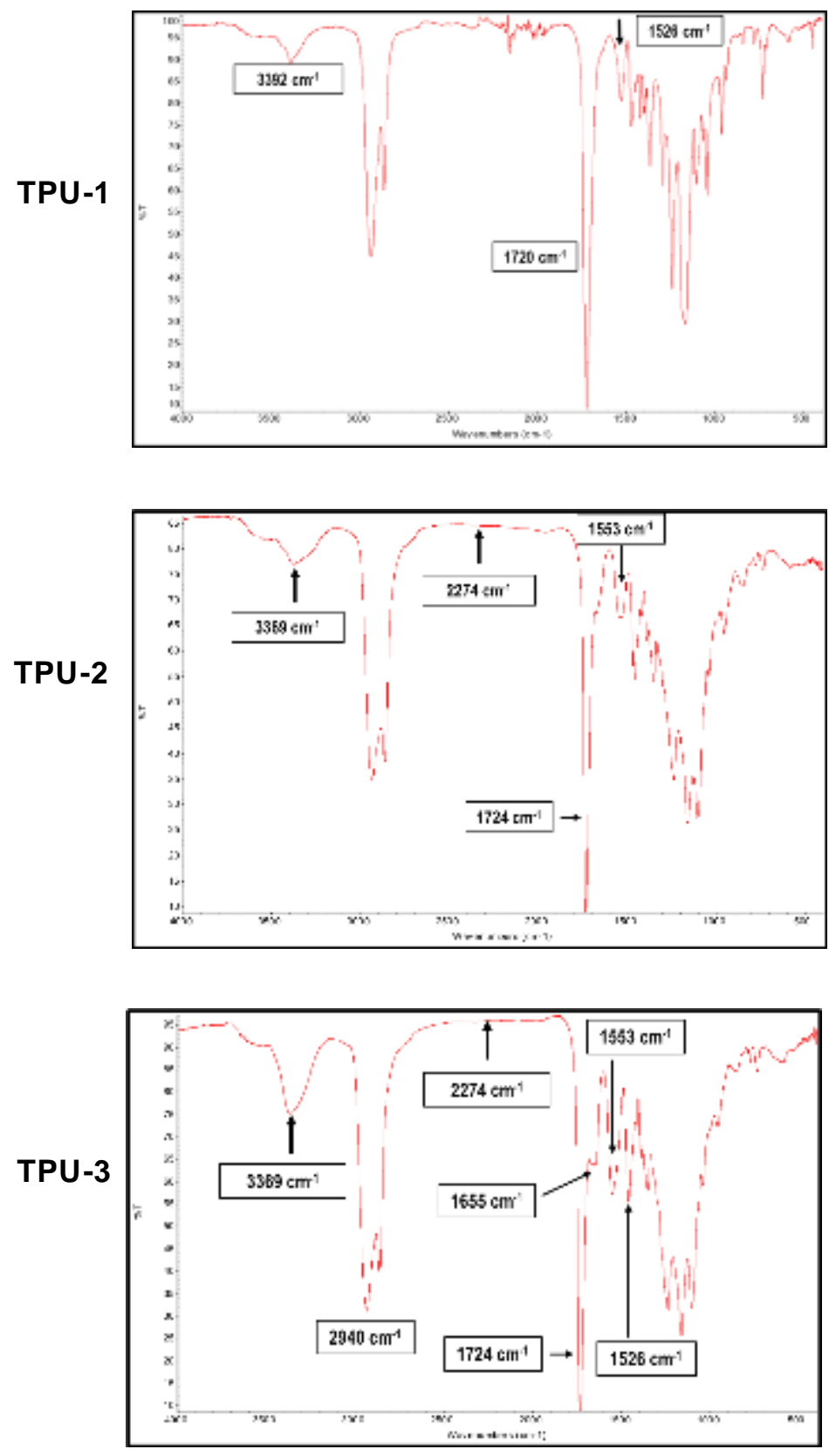

Figure S2-Sn. Chemical characterization of TPUs by FTIR scans. 

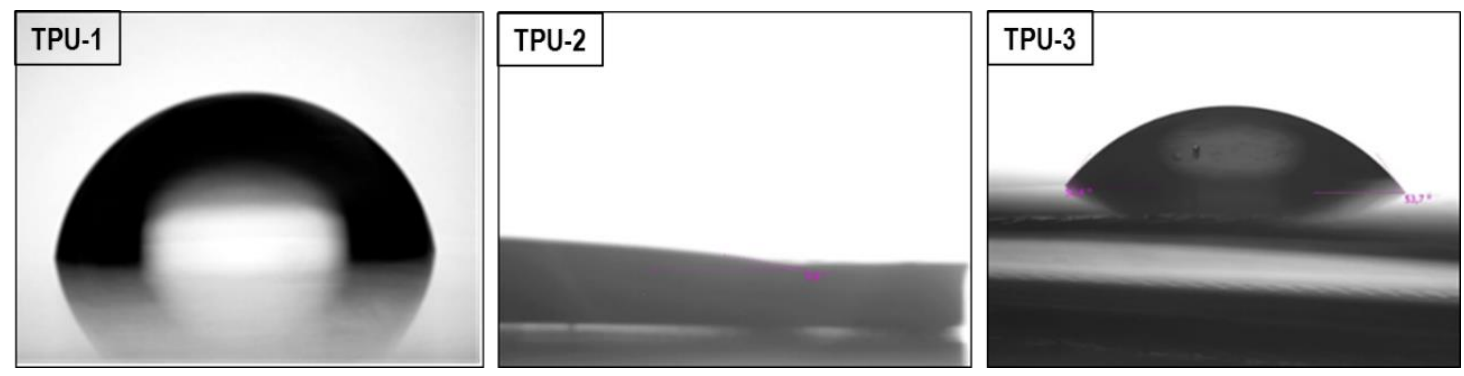

Figure S3-Sn. Images of water droplets recorded during static water contact angle measurements.
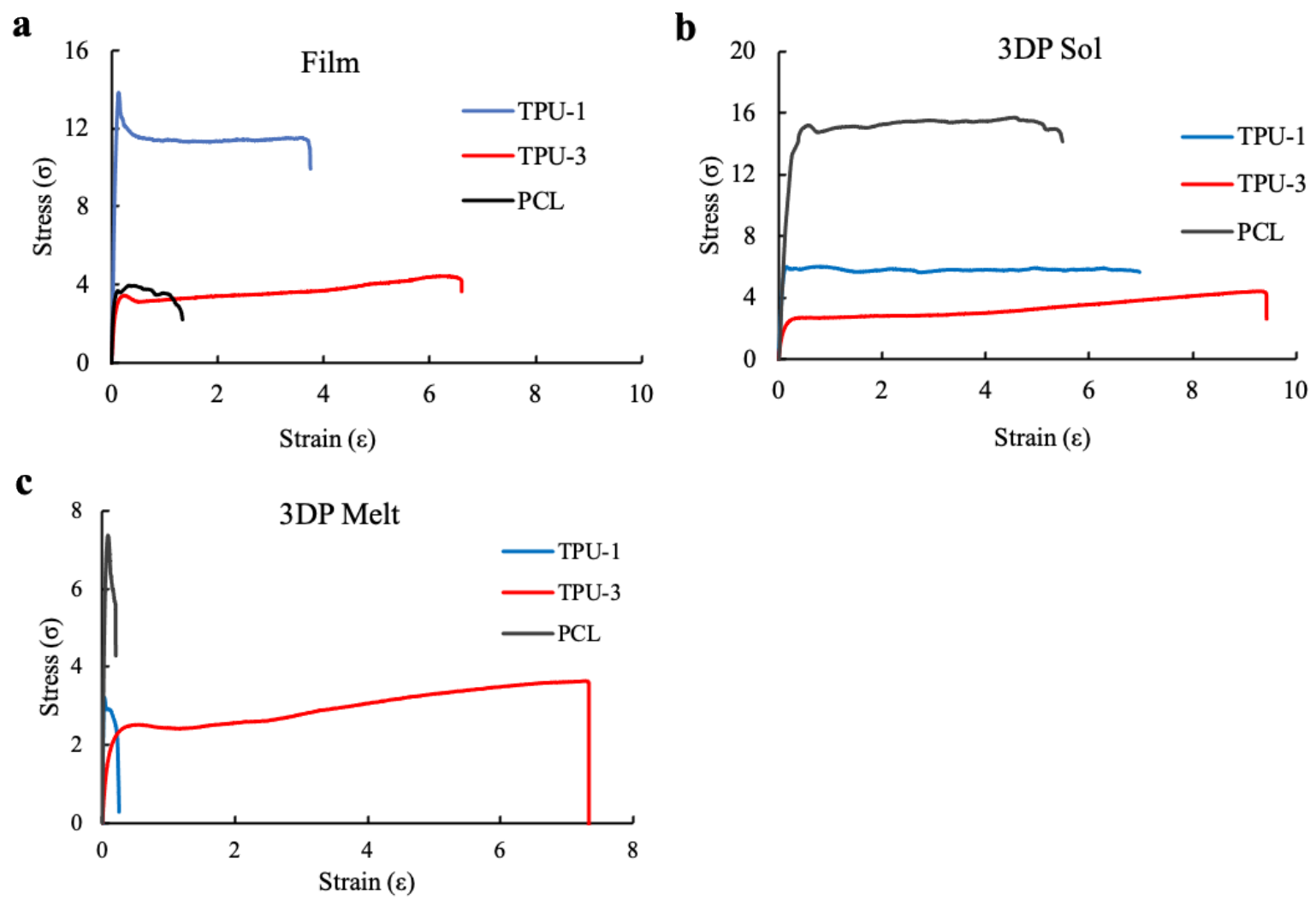

Figure S4-Sn. Stress-strain curves TPU-1 and TPU-3 with PCL as control. (a) Cast film, (b) 3D printed scaffold in solution state, (c) 3D printed scaffold in molten state. 
$\mathbf{a}$

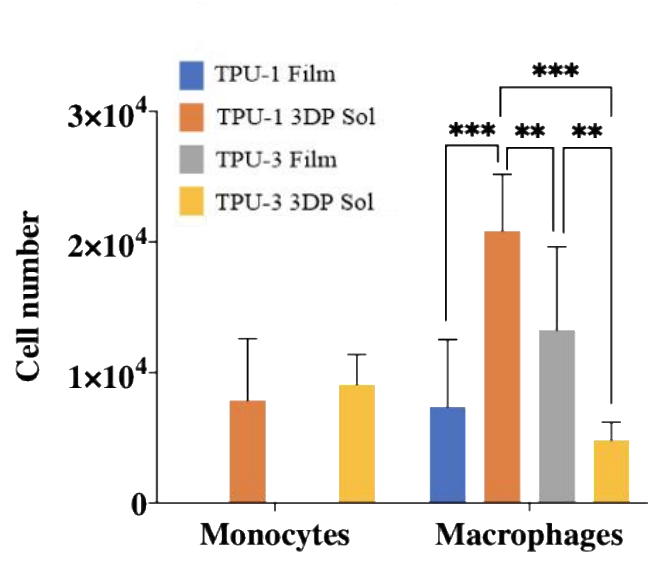

b

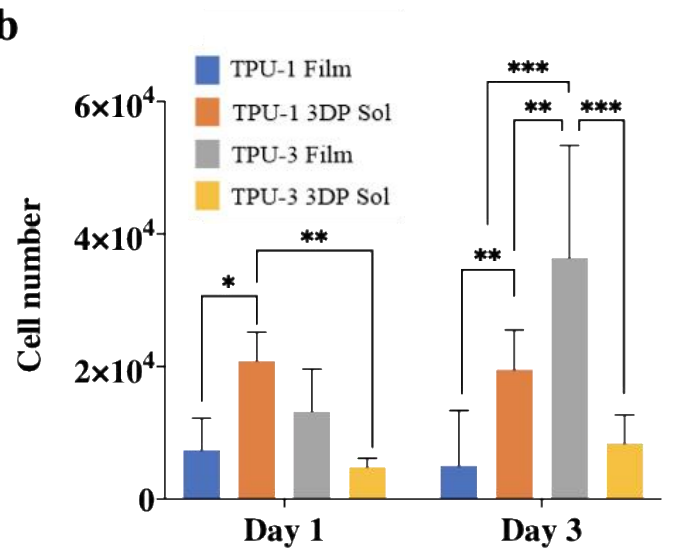

c
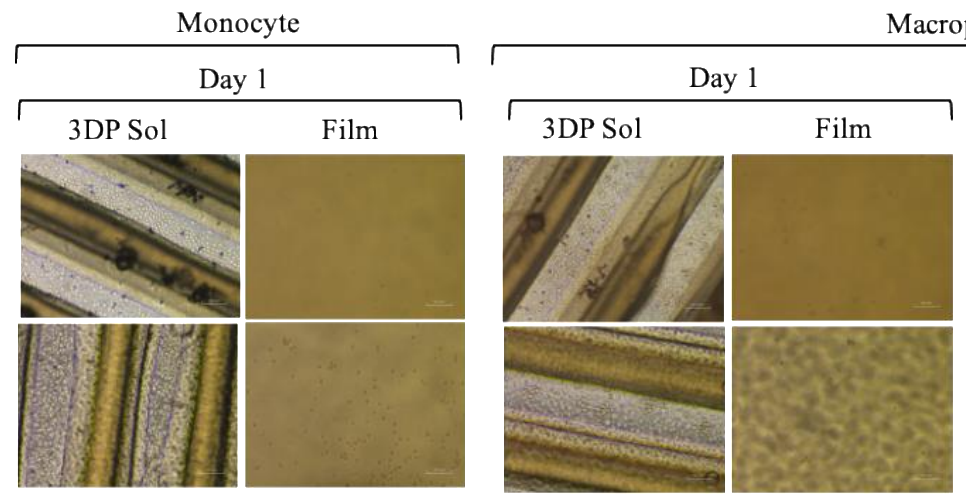

d

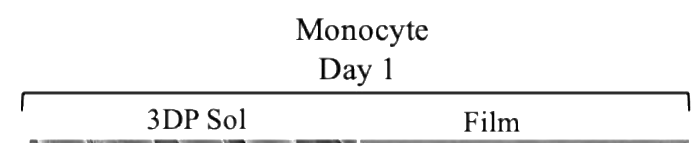

TPU-1

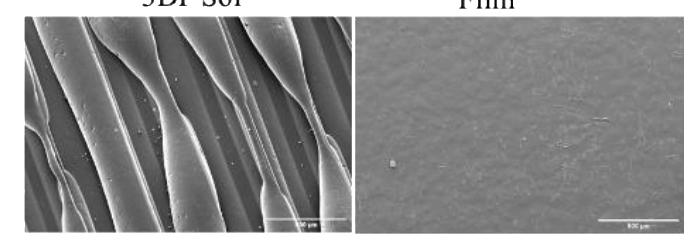

TPU-3

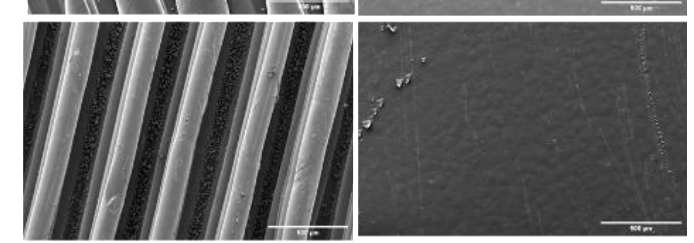

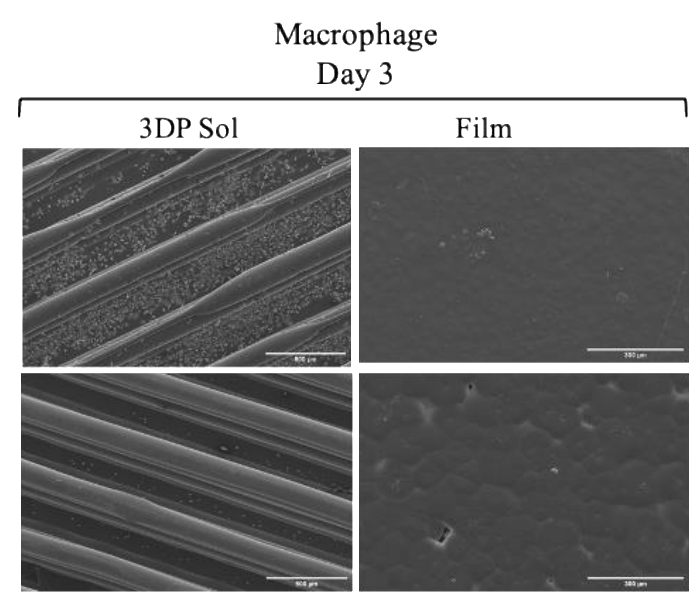

Figure S5-Sn. Metabolic activity of THP-1 (a) monocytes and macrophages on day 1, (b) macrophages on day 1 and day 3, seeded on TPU-1/TPU-3, 3D printed scaffolds/films. Data are average of three independent experiments $(* \mathrm{p}<0.05, * * \mathrm{p}<0.01$, *** $\mathrm{p}<0.001)$. (c) Phase contrast microscope images of THP-1 monocytes on day 1, THP-1 macrophages on day 1 and day 3, cultured on TPU-1/TPU-3, 3D printed scaffolds/films. Scale bar: $100 \mu \mathrm{m}$. (d) SEM micrographs of THP-1 monocytes on day 1 and THP-1 macrophages on day 3, cultured on TPU-1/TPU-3, 3D printed scaffolds/films. Scale bar: $100 \mu \mathrm{m}$. 

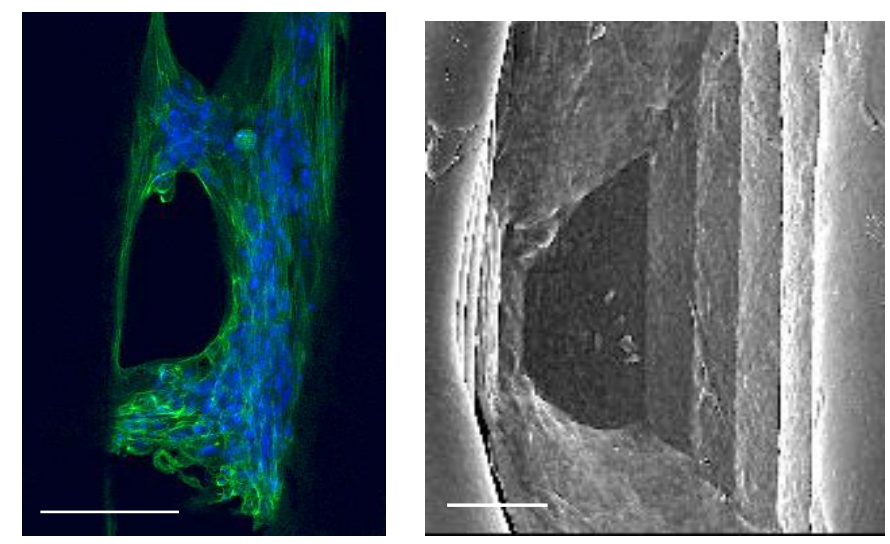

Figure S6-Sn. Verification of ASC attachment and spreading on 3D printed TPU-3 scaffold prior to in vivo study. Left image: Phalloidin/DAPI staining, Right Image: SEM micrograph. Scale bars: $250 \mu \mathrm{m}$.
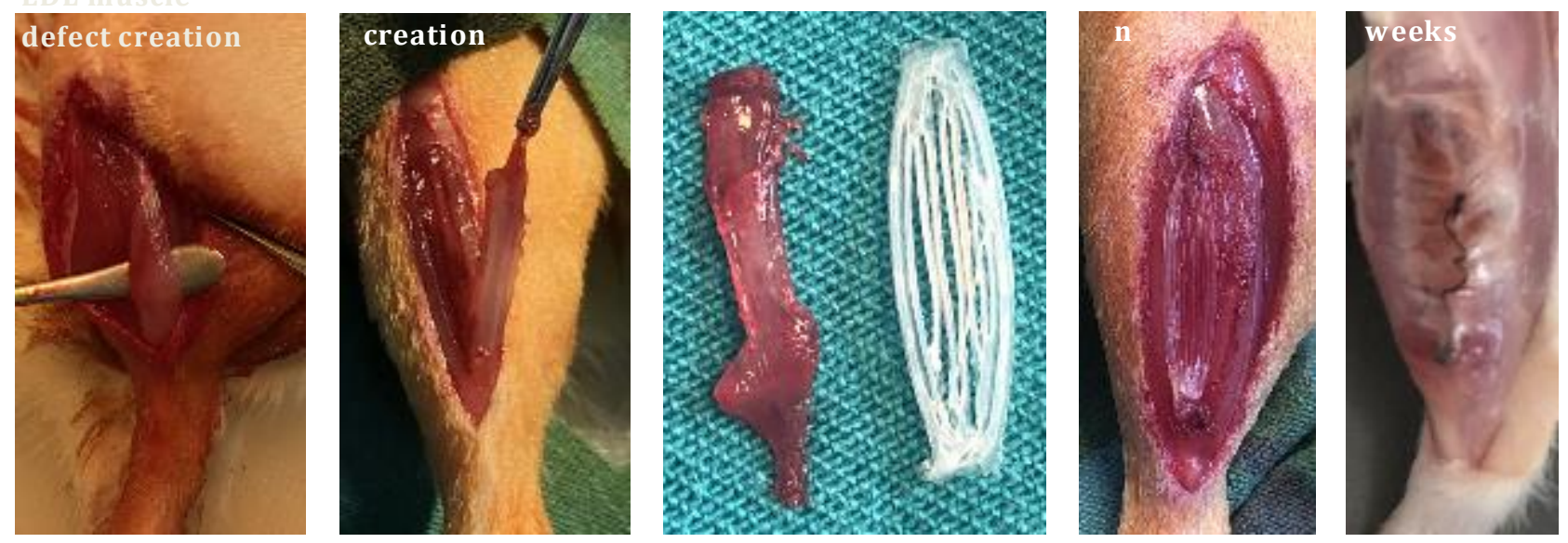

Figure S7-Sn. Rat tibialis anterior muscle was excised and implanted with 3D printed TPU-3 scaffold prepared to match the anatomical structure. 

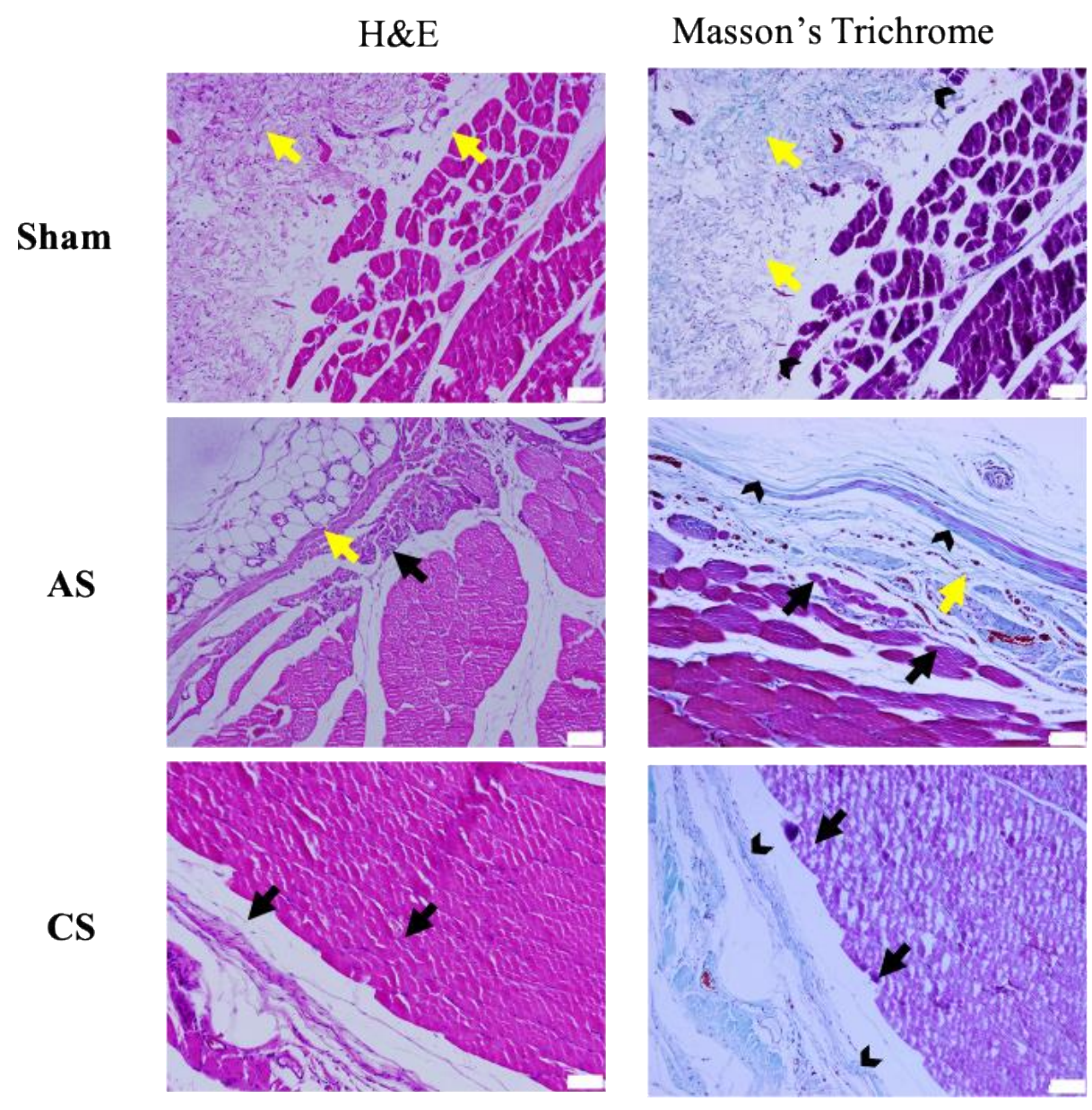

Figure S8-Sn. H\&E and Masson's Trichrome staining after 4 week post implantation for Empty, Acellular Scaffold (AS) and Cell-laden Scaffold (CS) groups in transversal sections, $10 \mathrm{x}$. Scale bar: $50 \mu \mathrm{m}$. In Empty group, blue staining of the collagen bundles (arrowhead) replacing the muscles together with the adipose cells (yellow arrow) in the defect area, Masson's Trichrome. In the acellular scaffold, Masson's Trichrome dye showing the blue staining connective tissue proliferation (arrowhead) and red staining proliferating myoblasts (arrow). Newly formed small myotubes (arrows) and inflammatory cells accompanied by connective tissue cells stained in blue (arrowhead) by Masson's trichrome staining in cell-laden scaffold groups. 

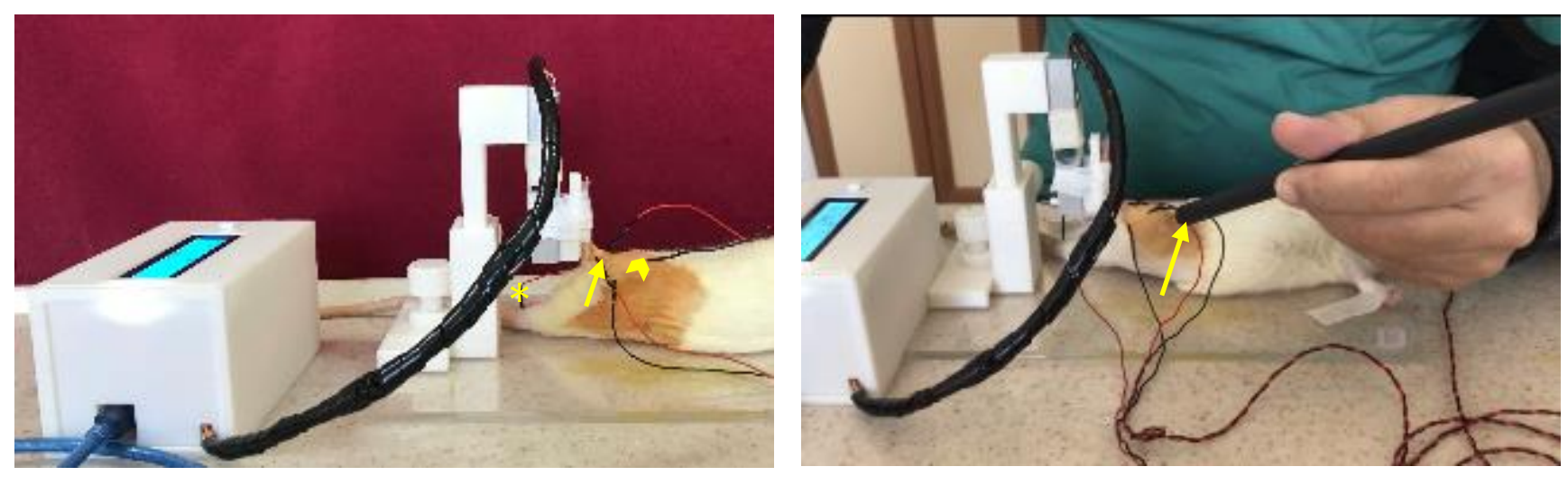

Figure S9-Sn. Custom made force transducer apparatus to assess the force generated by the regenerating muscle. a) Needle electrodes were placed in the middle of the tibialis anterior muscle (arrow), the Achilles tendon (arrowhead), and the tail of the rat (asterix) b) the peroneal nerve was stimulated using ADINSTRUMENTS animal nerve stimulation electrode (arrow). 